\title{
Evaluation of Solid-Solution Hardening in Several Binary Alloy Systems Using Diffusion Couples Combined with Nanoindentation
}

DOI: $10.1007 / \mathrm{s} 11661-017-4250-3$

(C) The Minerals, Metals \& Materials Society and ASM International 2017

\section{INTRODUCTION}

THE plastic flow resistance of a metal can be enhanced through various strategies like work hardening, grain boundary strengthening, solid-solution hardening and precipitation strengthening. Among these, solid-solution hardening ( $\mathrm{SSH}$ ) is perhaps the simplest and the most widely used method. Together with the construct of equilibrium phase diagrams and the theory of diffusion kinetics, the understanding of SSH behavior and mechanisms is essential to the development of materials with improved properties. As a result, SSH in binary solid-solution alloys has been a widely studied topic. ${ }^{[1-4]}$ Despite this, such studies have largely been limited to low-solute concentrations due to the requirement of only minor solute additions to strengthen structural alloys. However, with recent advances in the development of multiple-principle element alloys and

SOURABH B. KADAMBI is with the Department of Materials Engineering, Indian Institute of Science, Bangalore 560012, India, and also with the Department of Materials Science and Engineering, North Carolina State University, Raleigh, NC, 27695. V.D. DIVYA is with the Department of Materials Engineering, Indian Institute of Science, and also with Rolce-Royce India Private Limited, Manyata Embassy Business Park, Outer Ring Road, Bangalore 560045, India. U. RAMAMURTY is with the Department of Materials Engineering, Indian Institute of Science. Contact e-mail: ramu@materials.iisc.ernet.in

Manuscript submitted April 13, 2017.

Article published online July 27, 2017 the realization of their potential for structural applications, ${ }^{[5]}$ it has also become necessary to understand SSH at large solute concentrations.

The conventional method of obtaining SSH datastrength or hardness as a function of solute concentration - is through the synthesis and testing of one alloy with a specific composition at a time. However, this approach is not effective as it is time consuming and cost intensive. In addition, such an approach will be impractical in studying SSH in multicomponent alloys like high-entropy alloys which will require synthesis and testing of thousands of alloy compositions. Therefore, alternative experimental approaches that facilitate rapid assessment of SSH are required. In this context, the diffusion couple (DC) approach combined with smallscale testing methods such as nanoindentation and/or micropillar compression holds great promise. ${ }^{[6-14]} \mathrm{By}$ means of the DC method, a library of compositions is generated in the interdiffusion zones, and the compositional analysis along these zones is performed with a high degree of accuracy by employing techniques such as electron probe microanalysis (EPMA). The interdiffusion zones are then probed for hardness, $H$, by employing nanoindentation (NI), and $H$ as a function of solute concentration, $c$, is obtained. This approach is not only rapid but also requires possibly the minimum amount of material for synthesis and testing.

The DC-NI approach pioneered by Zhao ${ }^{[6-8,13]}$ has been used-for the determination of diffusion coefficients, to generate phase diagrams, and to obtain $\mathrm{H}-\mathrm{c}$ 
data in certain binary and ternary alloy systems. It has, however, only been used to a limited extent to study the SSH behavior. ${ }^{[9,11,13]}$ For example, Zhao ${ }^{[13]}$ and Divya et al. ${ }^{[11]}$ used the DC-NI method to obtain $H-c$ data in a few Ni-based binary and ternary alloy systems. However, these studies restricted their analyses to only qualitative comments on the SSH potency of various elements. The most significant work utilizing this method for analyzing SSH behavior is by Franke et al. ${ }^{[9]}$ - this study observed that the Labusch-type dependence, i.e., $H \propto c^{2 / 3}$, is obeyed in Ni-rich $\mathrm{Ni}-\mathrm{Fe}$, $\mathrm{Ni}-\mathrm{Mo}$, and Ni-Ta binaries and quantified the SSH behavior in terms of the SSH coefficient $k$.

In the present study, we extend the DC-NI approach to binary alloys of $\mathrm{Fe}$ containing $\mathrm{Ni}$ and $\mathrm{Co}$; $\mathrm{Pt}$ containing $\mathrm{Ni}$ and $\mathrm{Co}$; Co containing $\mathrm{Fe}, \mathrm{Pt}, \mathrm{Ni}$, and $\mathrm{Mo}$. We critically examine the compositional range - specifically large solute concentrations-over which the Labuschtype dependence of $H \propto c^{2 / 3}$ applies. We obtain the SSH coefficient $k$ by fitting experimental data to the Labusch's model and thus assess the SSH ability of the various solvent-solute combinations. We attempt to rationalize the $k$ values in terms of a misfit parameter that describes the atomic size and the elastic modulus differences between the solvent and solute elements and compare the obtained values of $k$ for $\mathrm{Ni}$ solid-solutions with the literature. We evaluate the reproducibility of SSH behavior obtained using the DC-Ni approach by comparing $k$ values (of $\mathrm{Ni}$ binaries containing $\mathrm{Fe}, \mathrm{Co}, \mathrm{Pt}$, and $\mathrm{Mo}$ ) obtained in this study with prior studies. We thus confirm the general applicability of the DC-NI approach to quantitatively study SSH.

\section{BACKGROUND}

A brief description of the SSH model used to analyze the $H-c$ data is presented here. The two major theories of SSH are due to Fleischer ${ }^{[2]}$ and Labusch, ${ }^{[15]}$ both of which evaluate the strengthening through statistical consideration of the interaction between dislocation and solute atoms. While the Fleischer model considers solute atoms as isolated pinning sites, each interacting with the dislocation line independently, the Labusch model considers the collective action of the solute atoms lying on the glide plane. Thus, the Labusch model is applicable to concentrated solid-solutions such as those examined in the present study, whereas the Fleischer model is only applicable at dilute concentrations $(c<1$ at. pct). ${ }^{[16]}$ Accordingly, the Labusch model is found to better describe the experimental data obtained in this study. As per the model, the change in shear stress on addition of solute atoms to the solvent lattice is directly proportional to $c^{2 / 3}$, and this relationship has been confirmed in a recent fully-analytical treatment of $\mathrm{SSH}^{[17]}$ Note that while the Labusch model is strictly valid for SSH close to $0 \mathrm{~K}$, it is generally found to be applicable at room temperature where atomic-size and modulus misfit effects dominate.

The Labusch expression for shear stress is reformulated to obtain an expression in terms of hardness using the Taylor factor and the Tabor factor for conversion from shear stress to uniaxial stress and from uniaxial stress to hardness, respectively, as:

$$
\Delta H=A \varepsilon^{4 / 3} c^{2 / 3}
$$

where $\Delta H$ is the change in $H$ of a pure solvent lattice resulting from the addition of solute; $\varepsilon$ is the combined misfit parameter that accounts for the contribution to $\Delta H$ arising from atomic size (or lattice parameter) and shear modulus, $G$, difference between the solvent and solute elements; $A \varepsilon^{4 / 3}$, which we will denote by $k$, represents the SSH coefficient or the hardening rate. $k$ is a constant for a specific solvent-solute combination and indicates the ability of the solute element to strengthen the solvent lattice. $k$ is obtained by fitting empirical data to the Labusch expression. In general, SSH results from elastic, chemical and electronic contributions associated with dislocation-solute interactions, and could also involve magnetic and ordering contributions in certain alloys. However, only elastic effects are considered here since elastic effects generally dominate. The elastic contribution to $\mathrm{SSH}$ is given by the misfit parameter, defined in Reference 18, as

$$
\varepsilon=\left(\varepsilon_{\mu}^{\prime 2}+\alpha^{2} \varepsilon_{a}^{2}\right)^{1 / 2}
$$

where $\varepsilon_{\mu}^{\prime}=\varepsilon_{\mu} /\left(1+\left|\varepsilon_{\mu}\right| / 2\right)$ is the modulus misfit parameter, and $\varepsilon_{a}$ is the atomic-size misfit parameter. For the purpose of this study, the parameters are defined as $\varepsilon_{\mu}=\Delta E / E_{\mathrm{PS}}$ and, from the linear size-factor defined in Reference $19, \varepsilon_{a}=\Delta r / r_{\mathrm{PS}} ; E_{\mathrm{PS}}$ and $r_{\mathrm{PS}}$ are the elastic modulus and the atomic radius of the pure solvent, respectively, and $\Delta E$ and $\Delta r$ are the differences in the respective quantities between the pure solvent and pure solute elements of the binary element combination. These quantities are obtained using pure element data from Reference 20. In evaluating $\Delta E$ and $\Delta r$, we assume a linear variation (with respect to concentration in at. pct) between the pure solvent and pure solute; this is the rule of mixtures approximation given by the Vegard's law. The value of $\alpha=16$ in the expression makes $\varepsilon_{a}$ the dominant factor in the evaluation of $\varepsilon$ and is typically used for FCC alloys to account for the interaction of solute atoms with edge dislocations; $\alpha=3$ is used for $\mathrm{BCC}$ alloys to account for the interaction of solute atoms with screw dislocations. ${ }^{[2]}$ Note, however, that the parameters are better evaluated by measuring the lattice parameter and elastic modulus variation with $c$; this approach is not used as data for all alloys studied here was not available in the literature.

\section{MATERIALS AND METHODS}

We examined SSH in the following binary combinations: Fe-Ni, Fe-Co, Pt-Ni, Pt-Co, Ni-Co, Ni-Mo and Co-Mo. Foils of $1 \mathrm{~mm}$ thickness of $\mathrm{Fe}, \mathrm{Ni}$ and $\mathrm{Co}$ with 99.95 pct purity and Mo with 99.99 pet purity were received from Alfa Aesar. Square pieces of $5 \mathrm{~mm} \times 5$ $\mathrm{mm}$ dimension were sliced from each element using low-speed saw. The cross sections were ground using a 
series of $\mathrm{SiC}$ grits and fine polished to a surface finish of $0.25 \mu \mathrm{m}$ using diamond paste. The samples were cleaned ultrasonically in ethanol and subsequently dried using hot air. Two different metal pieces were placed one above the other and clamped in a special fixture made of 316 steel while making sure that the pressure applied was minimum. A thin foil of Mo (or W) was used as a diffusion barrier between the samples and fixture to prevent any diffusion from the fixture material to the DCs. The DCs were then heat treated at relatively high temperatures to allow sufficient interdiffusion to occur between the two metals. Ni-Mo and Co-Mo were annealed at $1498 \mathrm{~K}\left(1225^{\circ} \mathrm{C}\right)$ for 9 hours; Fe-Ni and Fe-Co were annealed at $1473 \mathrm{~K}\left(120{ }^{\circ} \mathrm{C}\right)$ for 9 hours; $\mathrm{Ni}-\mathrm{Pt}, \mathrm{Ni}-\mathrm{Co}$ and Co-Pt were annealed at $1523 \mathrm{~K}$ $\left(1250{ }^{\circ} \mathrm{C}\right)$ for 25 hours. In all cases, the uncertainty in annealing temperature is within $\pm 5^{\circ} \mathrm{C}$. While the DCs of $\mathrm{Fe}-\mathrm{Ni}$, Fe-Co, Ni-Mo and Co-Mo (i.e., those containing $\mathrm{Fe}$ or $\mathrm{Mo}$ as one of the elements) were water-quenched to room temperature to retain their large solid solubility at high temperature, DCs of Ni-Pt, $\mathrm{Ni}-\mathrm{Co}$ and $\mathrm{Co}-\mathrm{Pt}$ were furnace cooled as they possess large solid solubility at room temperature. The DCs were mounted in Bakelite and cross sectioned using a low-speed diamond saw. Cross-sections were then ground and fine polished using $0.04 \mu \mathrm{m}$ colloidal silica suspension. After ultrasonic cleaning, the surfaces were examined using scanning electron microscopy (SEM Quanta 200) and the composition profiles across the interdiffusion zones were measured using electron probe microanalyzer (EPMA JEOL JXA-8530F).

Nanoindentations were performed across the interdiffusion zones of the DCs using a Hysitron TI 900 Triboindenter system equipped with a Berkovich diamond tip of $\sim 100 \mathrm{~nm}$ diameter. Loading and unloading rates of $2 \mathrm{mN} \mathrm{s}^{-1}$ and a peak load of $20 \mathrm{mN}$ with a hold time of 5 seconds at the peak load were employed. The indents were spaced at a sufficient distance to avoid any overlap of their stress/strain fields. To obtain good spatial resolution combined with the necessary indent spacing, indentations were placed along an imaginary line $\sim 45 \mathrm{deg}$ to the DC junction. An SEM image of the DC and the indentations corresponding to the Co-Fe DC is shown in Figure 1(a) as representative of all other DCs. To ensure accurate measurement of properties, area function calibration of the tip was carried out prior to testing using a single crystal fused quartz standard with a specified modulus of $69.4 \mathrm{GPa}$. Local compositions along the interdiffusion zones were measured using EPMA and mapped to the hardness profile as shown for Co-Fe DC shown in Figure 1(b) (refer to the Supplementary Material for data corresponding to the other DCs).

\section{RESULTS AND DISCUSSION}

The $H$ vs $c$ plots of all binary alloys examined in this work are shown in Figures 2(a) and (b). In Ni-Mo and Co-Mo, very high hardness values of 11 and $16 \mathrm{GPa}$ are obtained near the equiatomic composition. These compositions are identified as intermetallic phases from the discontinuity in the concentration-distance profiles
(Figures S1(d) and (e)) as characterized in prior studies of Divya et al. ${ }^{[10,21]}$ We exclude these data points from our analysis as they do not represent solid-solutions. We also exclude data corresponding to Mo-rich end of the DCs as Mo shows limited solid solubility of less than 1 at. pct. For analysis using the Labusch model of Eq. [1], we plot $H$ vs $c$ data in terms of enhancement in hardness, $\Delta H$, against $c^{2 / 3}$; accordingly, we will refer to $\Delta H$ vs $c^{2 / 3}$ plots as Labusch plots here afterwards.

\section{A. Fe-Co and Fe-Ni Binary Alloys}

The Labusch plots of Fe-Co and Fe-Ni, presented in Figure 3 , show that $\Delta H$ increases bilinearly with $c^{2 / 3}$. The SSH coefficients $k$ are obtained by fitting the linear portions of the data to the Labusch expression of Eq. [1]. Corresponding to concentrations less than $\sim 9$ at. pet $\mathrm{Co}$ and $\sim 15$ at. pet $\mathrm{Ni}$, the $k$ values obtained for $\mathrm{Fe}-\mathrm{Co}$ and $\mathrm{Fe}-\mathrm{Ni}$ (44 and $40 \mathrm{MPa} /$ at. $\mathrm{pct}^{2 / 3}$, respectively) indicate similar but weak SSH effect of these solutes in BCC Fe. This result is reasonable since both $\mathrm{Co}$ and $\mathrm{Ni}$ have comparable $r$ and $E$ values with $\mathrm{Fe}$ (the atomic-size, $\Delta r$, and the elastic modulus, $\Delta E$, difference for the various solvent-solute combinations are presented in Table I). Estimation of the combined misfit parameter $\varepsilon$, from Eq. [2], gives relatively small values (0.05 and 0.09 for $\mathrm{Fe}-\mathrm{Co}$ and $\mathrm{Fe}-\mathrm{Ni}$, respectively), which, along with the negligible lattice parameter variations of $\mathrm{Co}$ and $\mathrm{Ni}$ in $\mathrm{BCC} \mathrm{Fe}^{[22]}$ support the results. The observed $\mathrm{SSH}$ behavior of $\mathrm{Fe}-\mathrm{Co}$ is in agreement with a number of studies, with the most notable being that of Leslie et al. ${ }^{[23]}$ which finds that $\mathrm{Co}$ is an ineffective strengthening solute in $\mathrm{BCC} \mathrm{Fe}$. Comparison of the observed $\mathrm{SSH}$ in $\mathrm{Fe}-\mathrm{Ni}$, however, is not straightforward (and hence not undertaken here), with significantly different $\mathrm{SSH}$ and solid-solution softening behaviors reported ${ }^{[23]}$ under different testing conditions of strain rate and temperature.

The $k$ values obtained for $\mathrm{Fe}-\mathrm{Co}$ and $\mathrm{Fe}-\mathrm{Ni}$ are considerably higher at large solute concentrations. This is inferred to be a result of the change in crystal structure of the solid-solution phase from BCC at low $c$ to FCC at large $c$, rather than due to a change in the SSH ability of the solutes with $c$. Fe-Co and Fe-Ni DCs, which were annealed at $1473 \mathrm{~K}\left(1200{ }^{\circ} \mathrm{C}\right)$, are completely miscible in the FCC region of the phase diagrams at this temperature. On quenching the DCs to room temperature, the system is implicitly assumed to retain the large solute solubility and the FCC phase. However, Fe and Fe-rich alloys do not retain the high-temperature FCC phase but transform to a (supersaturated) BCC phase through diffusionless transformation. It is known from studies $^{[26,27]}$ employing similar heat treatment on $\mathrm{Fe}$ alloys that alloys with $c<25$ at. pet $\mathrm{Ni}$ transform to $\mathrm{BCC}$, while those with $c>28$ at. pct $\mathrm{Ni}$ retain their high-temperature FCC phase. Importantly, this transition correlates well with $\sim 15$ at. pet $\mathrm{Ni}$ concentration at which the two linear responses intersect in Figure 3. Since the addition of $\mathrm{Co}$, like that of $\mathrm{Ni}$, stabilizes the FCC phase of Fe-Co, the above inference on BCC to FCC transition with $c$ is also applicable to the $\mathrm{Fe}-\mathrm{Co}$ SSH data. 
An estimate of $H$ of FCC Fe $(1.18 \mathrm{GPa})$ is obtained by extrapolating the linear fit of FCC Fe-Ni to $c=0$. This value is lower than the average $H$ value obtained for BCC Fe (1.55 GPa). Since an FCC phase is expected to be softer than the $\mathrm{BCC}$ phase due to the presence of a larger number of slip systems in the former, the above observation is consistent with our inference of $\mathrm{BCC}$ and $\mathrm{FCC}$ regions. While the $k$ values for $\mathrm{BCC} \mathrm{Fe}-\mathrm{Co}$ and $\mathrm{Fe}-\mathrm{Ni}$ are similar, that for $\mathrm{FCC} \mathrm{Fe}-\mathrm{Co}$ is seen to be significantly higher than FCC Fe-Ni. Elastic interactions alone, however, do not explain the difference between the $k$ values of FCC $\mathrm{Fe}-\mathrm{Co}$ and $\mathrm{Fe}-\mathrm{Ni}$ since the calculated $\varepsilon$ values (Table I) are similar. A probable explanation for this difference then is the contribution of chemical or slip misfit effect to SSH. This effect has been incorporated ${ }^{[28]}$ to the Labusch model using the slip misfit parameter: $\varepsilon_{s}$ (which is conceptually similar to $\varepsilon_{a}$ and $\varepsilon_{\mu}$ ) given by $\frac{1}{\gamma} \frac{\mathrm{d} \gamma}{\mathrm{d}_{c}}$, where, $\gamma$ is the stacking fault energy (SFE). Since the SFE of FCC Fe decreases considerably with $\mathrm{Co}$ addition, ${ }^{[29]}$ the slip misfit parameter can be expected to contribute to the high value of $k$.

\section{B. Pt-Co and Pt-Ni Binary Alloys}

The Labusch plots for Pt-Ni and Pt-Co are shown in Figure 4. For Pt-Ni, the hardness data (until its maximum value) is described by a single straight line; we find the same to be true for Ni-Pt solid-solution (shown in Figure 5). This indicates an excellent agreement of the experimental data with the Labusch model. Ni-Pt exhibits isomorphism or complete miscibility and its FCC phase is retained to room temperature on cooling the DC. A strong $\mathrm{SSH}$ of $\mathrm{Pt}(H=1.41 \mathrm{GPa})$ is obtained on addition of $\mathrm{Ni}$, with $k=184 \mathrm{MPa}$ at. $\mathrm{pct}^{2 / 3}$; also, the SSH coefficient of $\mathrm{Ni}(H=1.82 \mathrm{GPa})$ on addition of $\mathrm{Pt}$ is given by $k=222 \mathrm{MPa} /$ at. $\mathrm{pct}^{2 / 3}$. Note here that the difference between hardness of the pure elements $\mathrm{Pt}$ and $\mathrm{Ni}$ and that between the $k$ values of $\mathrm{Pt}-\mathrm{Ni}$ and $\mathrm{Ni}-\mathrm{Pt}$ leads to the maximum value in hardness to occur at a non-equiatomic composition of 64 at. pet $\mathrm{Ni}$.

Since the Pt-Co DC was cooled at a slow rate from the diffusion annealing temperature, the ordered intermetallic phases are likely to have formed between 15 and 40 at. pct $\mathrm{Co}$ as per the equilibrium phase diagram. Coinciding with this compositional range, a marked increase in $\Delta H$ beyond $\sim 16$ at. pct Co with nonlinear dependence with $\mathrm{c}^{2 / 3}$, and high hardness values characteristic of the ordered intermetallics are observed. Thus, compositions below $\sim 16$ at. pct $\mathrm{Co}$ are considered as solid-solutions, and the $k$ value corresponding to this region is determined by the slope to the linear fit as $77 \mathrm{MPa} /$ at. pct $^{2 / 3}$.

Based on the SSH coefficients obtained, $\mathrm{Ni}$ and $\mathrm{Co}$ can be characterized to exhibit moderate SSH ability in $\mathrm{Pt}$, with $\mathrm{Ni}$ being more potent due its relatively large $\varepsilon$ value (Table I). This assessment is consistent with the work of Murakami et al. ${ }^{[30]}$ on the SSH behavior of 25 solutes in binary $\mathrm{Pt}$ solid-solution. Based on the size-misfit and complete miscibility, $\mathrm{Ni}$ and $\mathrm{Co}$ were found to be moderate SSH solutes in Pt. In a study using the DC-NI combinatorial approach, Zhao ${ }^{[13]}$ presents the $H-c$ data for Pt-Ni. Converting these data to the Labusch form, we obtain the $k$ value from linear fit as $350 \mathrm{MPa} /$ at. pct $^{2 / 3}$, which is nearly twice that obtained from our study. Although the reason for this discrepancy is not known, the difference in heat-treatment conditions could be a possibility [our Pt-Ni DC was slow cooled from $1523 \mathrm{~K}\left(1250^{\circ} \mathrm{C}\right)$, while that of $\mathrm{Zhao}^{[13]}$ was quenched from $\left.1173 \mathrm{~K}\left(900{ }^{\circ} \mathrm{C}\right)\right]$.

\section{Ni-Based Binary Alloys}

The Labusch plots for Ni-Mo, Ni-Pt, Ni-Fe, and $\mathrm{Ni}-\mathrm{Co}$ are shown in Figure 5. It is seen that $\mathrm{Mo}$ contributes significantly to $\mathrm{SSH}$ of $\mathrm{Ni}-10$ at. pet $\mathrm{Mo}$ addition results in nearly $2 \mathrm{GPa}$ increase in $H$, doubling the hardness of pure $\mathrm{Ni}$ (the average $H$ value of pure $\mathrm{Ni}$ from multiple nanoindentations on all Ni-based DCs is $1.82 \mathrm{GPa})$. In contrast, Co contributes insignificantly, with 10 at. pct addition, for instance, resulting in negligible hardening, while $\mathrm{Pt}$ and $\mathrm{Fe}$ provide moderate hardening. These observations can be rationalized using the elastic misfit and interaction parameters. For example, from $\Delta r$ and $\Delta E$ values in Table I, $\varepsilon$ for Ni-Mo $(=1.98)$ is obtained as significantly greater than that for $\mathrm{Ni}-\mathrm{Co}(=0.14)$, leading to a prediction of strong SSH ability of Mo and negligible SSH ability of Co. These are in qualitative agreement with the experimental results. The observed SSH is also in good agreement with the literature $e^{[9,31-33]}$ on macroscopic mechanical testing. The $k$ values of 411 and $11 \mathrm{MPa} / \mathrm{at} . \mathrm{pct}^{2 / 3}$ for $\mathrm{Ni}-\mathrm{Mo}$ and $\mathrm{Ni}-\mathrm{Co}$, respectively, can be converted to the SSH coefficient $K$ of the yield stress form of the Labusch model - using the Taylor and Tabor conversion factors (approximately 3 for both) yields estimates of $K$ for $\mathrm{Ni}-\mathrm{Mo}$ and $\mathrm{Ni}-\mathrm{Co}$ as 986 and $26 \mathrm{MPa} /(\text { at. frac. })^{2 / 3}$, respectively. These values compare well with 1015 and $39.4 \mathrm{MPa} /(\text { at. frac. })^{1 / 2}$ obtained by fitting the Fleischer model to compressive flow stress data. ${ }^{[32,33]}$ In addition, an excellent agreement is found between $k$ for $\mathrm{Ni}-\mathrm{Pt}$ $\left(222 \mathrm{MPa} /\right.$ at. $\left.\mathrm{pct}^{2 / 3}\right)$ obtained in this study and that $\left(190 \mathrm{MPa} /\right.$ at. pct $\left.^{2 / 3}\right)$ obtained by analyzing the data presented by Zhao. ${ }^{[13]}$

Based on the heat-treatment condition employed and the equilibrium phase diagrams, all compositions at the Ni-rich end of the DCs are inferred to correspond to FCC solid-solutions. In Ni-Fe, a bilinear dependence of $\Delta H$ with $c^{2 / 3}$ is observed. For $c>3$ at. pct $\mathrm{Fe}$, a comparatively high $k$ value of $141 \mathrm{MPa} / \mathrm{at} \mathrm{pct}^{2 / 3}$ is obtained, which compares reasonably well with $110 \mathrm{MPa} /$ at. $\mathrm{pct}^{2 / 3}$ (for $c$ between 0 and $\sim 30$ at. pct) reported by Durst et al. ${ }^{[24]}$ At low concentrations $(c<3$ at. pct $\mathrm{Fe})$, however, we find weak SSH with $k=11 \mathrm{MPa} /$ at. $\mathrm{pct}^{2 / 3}$. Interestingly, on analyzing $\mathrm{Ni}-\mathrm{Fe}$ data of, ${ }^{[24]}$ we find that for $c<3$ at. pct, the Labusch fit yields a low value of $40 \mathrm{MPa} /$ at. pct ${ }^{2 / 3}$ (see Figure S2 in Supplementary Material) - taking into account of the large scatter in the data, this value compares well with that obtained in the present study. In addition, these results are qualitatively consistent with the compressive flow stress data of Mishima et al. ${ }^{[31]}$ In Reference 31 the flow stress of $\sim 100 \mathrm{MPa}$ 


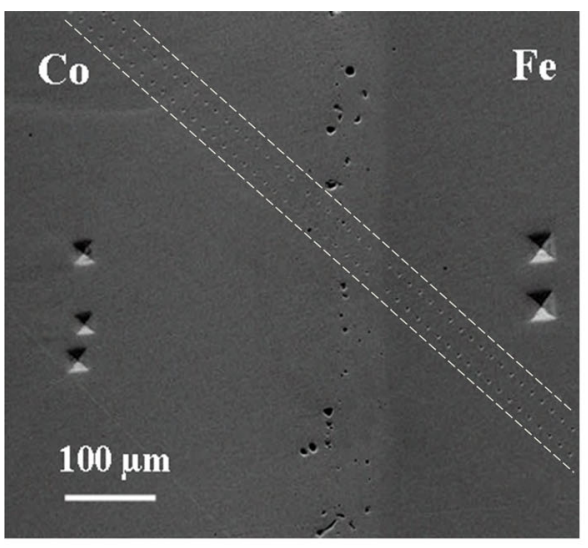

(a)

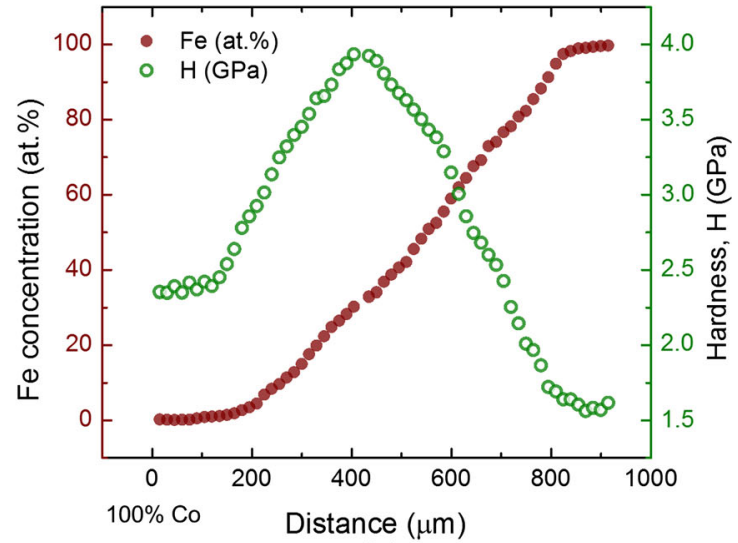

(b)

Fig. 1-(a) SEM image of Co-Fe diffusion couple (DC). The two parallel dotted lines (enclosed within the marked dashed lines) are the residual imprints of nanoindentations carried out in the pure Fe, pure Co regions, and across the interdiffusion zone of the DC. (b) Hardness, $H$, obtained from nanoindentations in (a), and the concentrations, $c$, at corresponding locations are plotted against the distance along the DC.

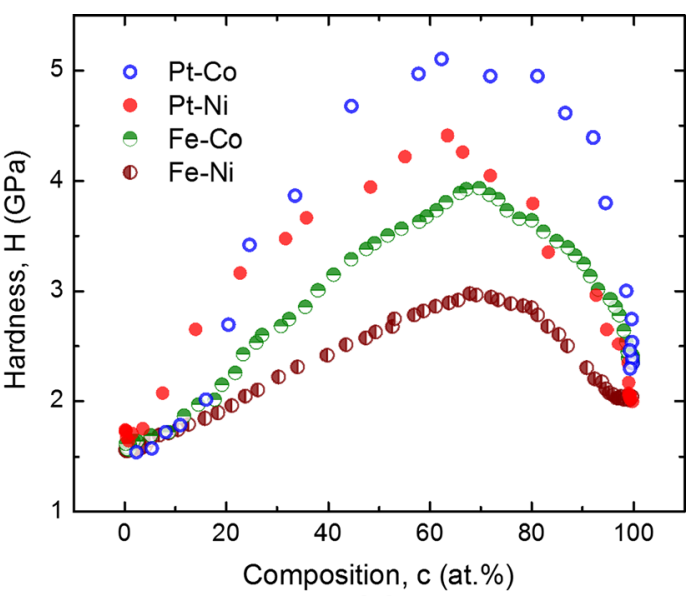

(a)

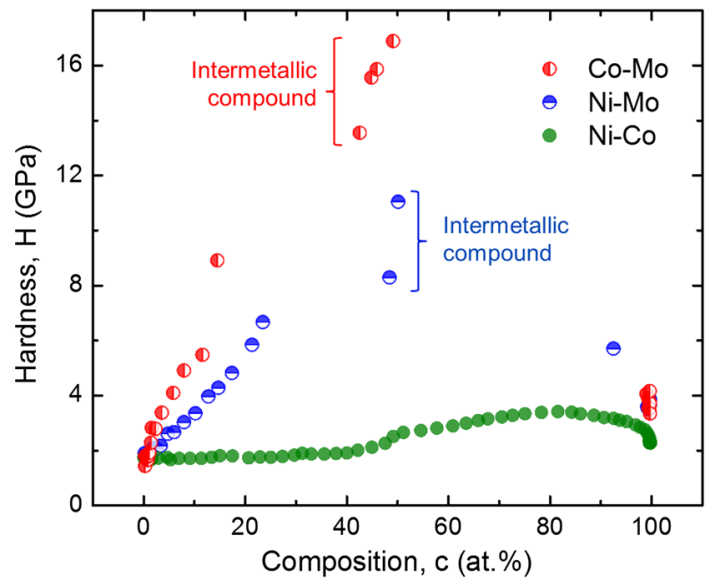

(b)

Fig. 2-Hardness-concentration, $H$ vs $c$, profiles for the various binary alloy combinations: (a) Pt-Co, Pt-Ni, Fe-Co, and Fe-Ni; (b) Co-Mo, Ni-Mo, and Ni-Co. The profiles are obtained by mapping $H$, obtained from nanoindentation at various locations along the interdiffusion zone, to $c$ measured at corresponding locations using EPMA.

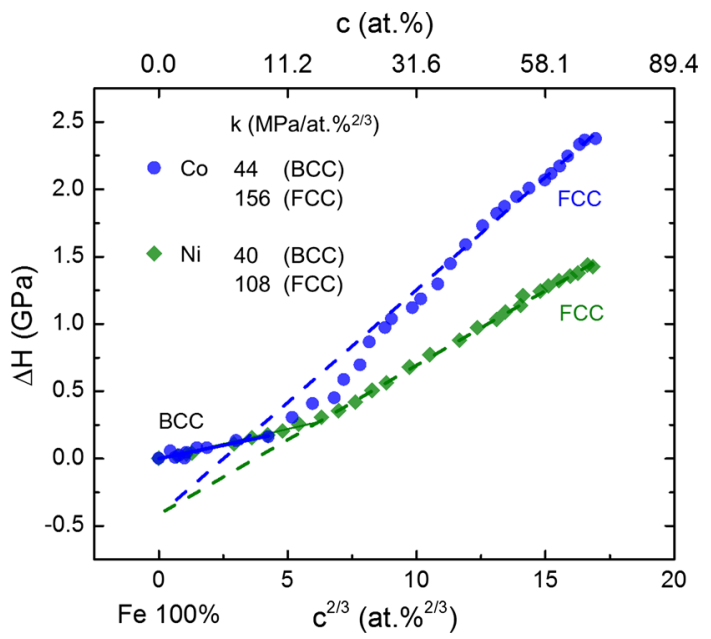

Fig. 3 - Changes in hardness, $\Delta H$, against solute concentration, $c^{2 / 3}$, corresponding to $\mathrm{Fe}-\mathrm{Co}$ and $\mathrm{Fe}-\mathrm{Ni}$. Dashed lines are linear fits to the solid-solution regions of $\mathrm{BCC}$ and FCC ( $c$ ranges used for fit is given in Table I). The linear fit to $\mathrm{FCC} \mathrm{Fe}-\mathrm{Ni}$ is extrapolated to $c=0$ to obtain an estimate of $H$ of $\mathrm{FCC} \mathrm{Fe}$; this value is used to choose the $c$ range for fitting to FCC Fe-Co. Values of SSH coefficients $k$, which are the slopes of linear fits, are presented within.

of pure Ni was retained on addition of $\sim 2$ at. pet Fe and increased to $\sim 150 \mathrm{MPa}$ for an 8 at. pet Fe addition. While the low value of $k$ obtained at low concentrations of $\mathrm{Fe}$ is expected considering only elastic interactions, the high value of $k$ above $\sim 3$ at. pct Fe must be due to additional nonelastic contributions.

In Ni-Co, a steep increase in hardness is observed beyond $\sim 40$ at. pet Co. Any phase transformation or intermetallic formation is not expected in this compositional region. A probable explanation, therefore, is ascribed to the contribution to $\mathrm{SSH}$ from stacking faults. Co has a significantly low SFE $\left(\sim 10 \mathrm{~mJ} / \mathrm{m}^{2}\right.$ for FCC $\mathrm{Co}^{[34]}$, and its addition to Ni lowers the SFE of the alloy. This results in an increased occurrence of SFs in the alloy during deformation, leading to additional strengthening through the suppression of cross slip-mediated deforma- 


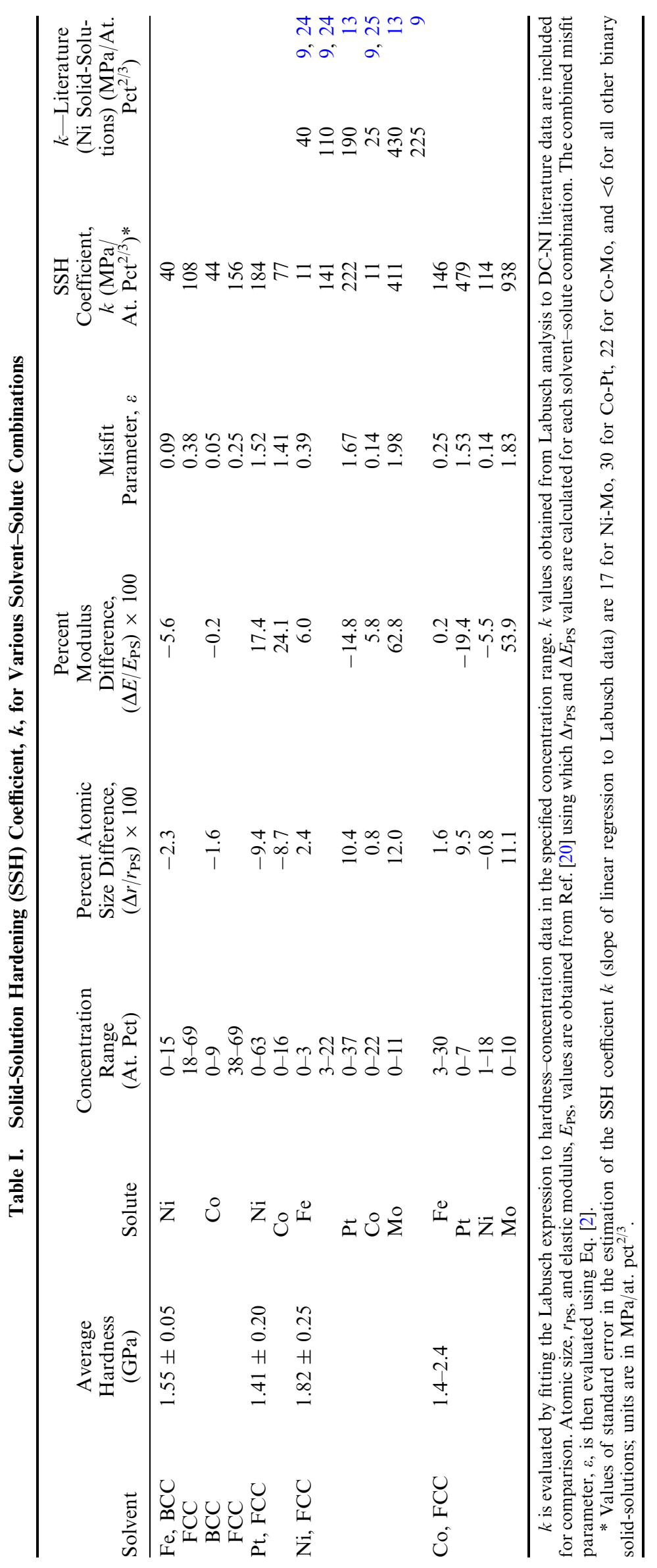




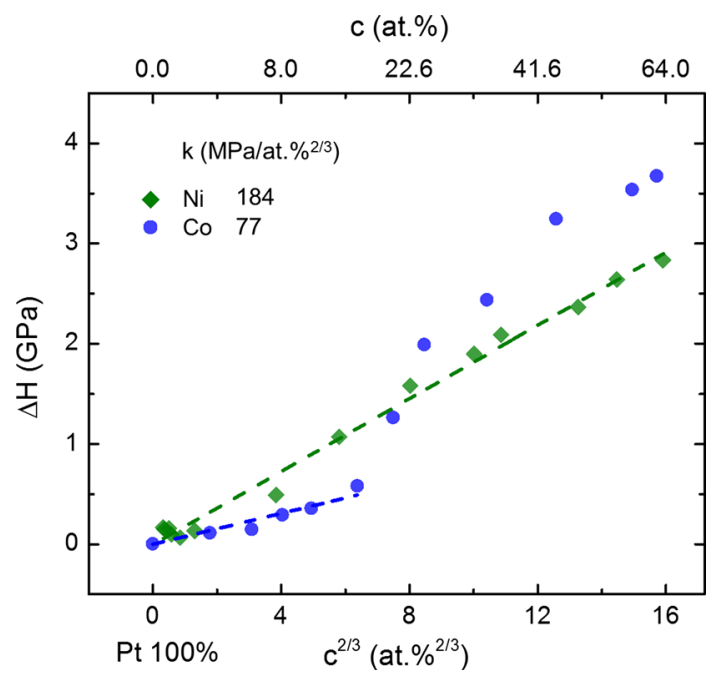

Fig. 4 Changes in hardness, $\Delta H$, against solute concentration, $c^{2 / 3}$, corresponding to Pt-Co and Pt-Ni. Dashed lines are linear fits to the solid-solution regions ( $c$ ranges used for fit are given in Table I). Values of SSH coefficients $k$, which are the slopes of linear fits, are presented.

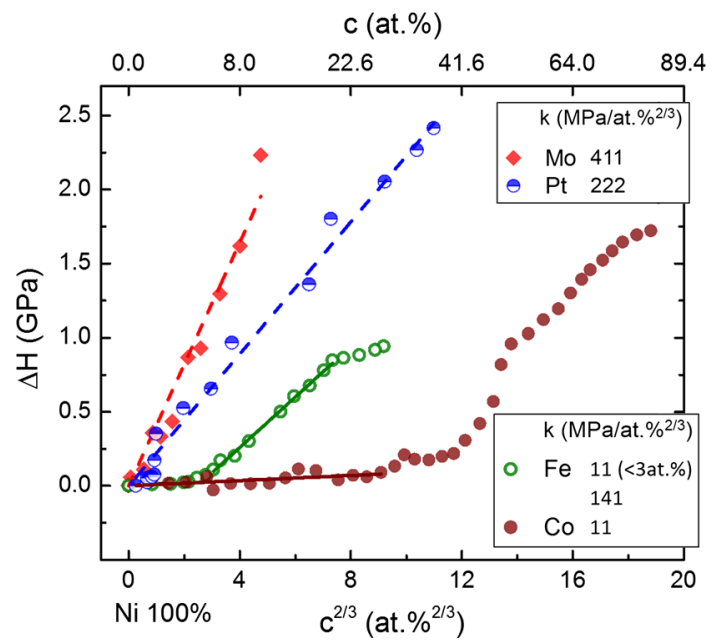

Fig. 5-Changes in hardness, $\Delta H$, against solute concentration, $c^{2 / 3}$, corresponding to $\mathrm{Ni}-\mathrm{Mo}$, Ni-Pt, Ni-Fe, and $\mathrm{Ni}-\mathrm{Co}$ are plotted until the maximal value in $\Delta H$. Dashed lines are linear fits to the solid-solution regions ( $c$ ranges used for fit are given in Table I). Values of SSH coefficients $k$, which are the slopes of linear fits, are presented within.

tion. This claim is substantiated from the observation of $\mathrm{SF}$ densities in deformed Ni-Co alloys by Delehouzee et al. ${ }^{[35]}$ It was found that a mere twofold increase in SF density occurred by increasing Co from 0 to 40 at. pct, whereas, a steep fivefold increase resulted upon increasing further Co from 40 to 70 at. pct. While this is consistent with the observed trends in $H$ of the present study, it remains to be seen whether similar densities of SFs form during nanoindentation deformation.

\section{Co-Based Binary Alloys}

The hardness obtained from the Co-rich end of $\mathrm{Co}-\mathrm{Fe}$ and $\mathrm{Co}-\mathrm{Ni} \mathrm{DCs}$ showed considerable scatter ranging

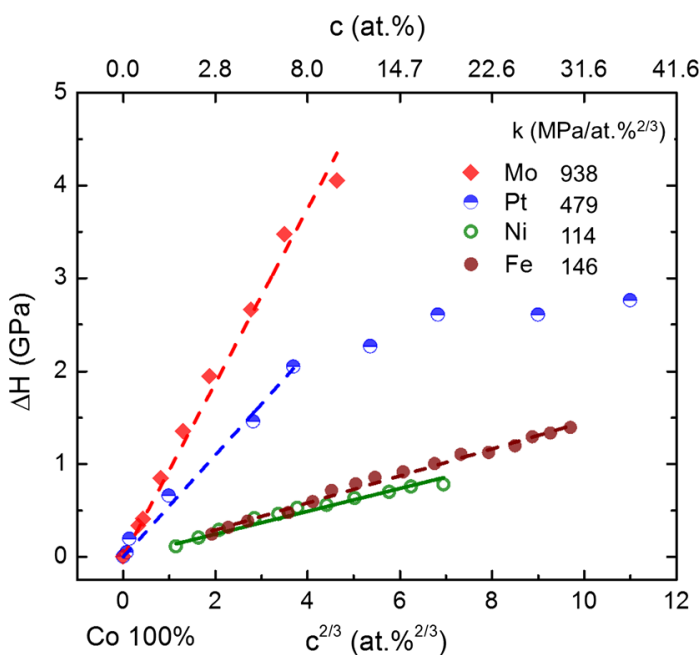

Fig. 6 - Changes in hardness, $\Delta H$, against solute concentration, $c^{2 / 3}$, corresponding to Co-Mo, Co-Pt, Co-Ni, and Co-Fe DCs are plotted against $c^{2 / 3}$ until maximum in $\Delta H$. Dashed lines are linear fits to the solid-solution regions ( $c$ ranges used for fit are given in Table I). Values of SSH coefficients $k$, which are the slopes of linear fits, are presented for FCC Co solid-solutions.

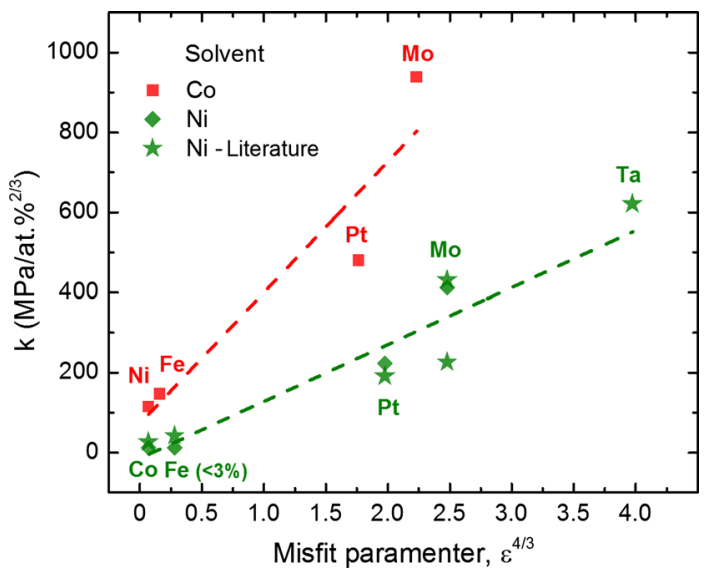

Fig. 7-Solid-solution hardening coefficients, $k$, for FCC Ni and Co solid-solutions are plotted against the elastic misfit parameter, $\varepsilon^{4 / 3} \cdot \varepsilon$ is evaluated from Eq. [2] using atomic radius and elastic modulus values from. ${ }^{[20]}$ Also represented are $k$ values obtained from the Labusch analysis (see Supplementary Material) to data taken from the literature: Ni-Ta $,{ }^{\left[{ }^{1]}\right.} \mathrm{Ni}-\mathrm{Mo},{ }^{[9,13]} \mathrm{Ni}-\mathrm{Fe},{ }^{[9,24]} \mathrm{Ni}-\mathrm{Pt},{ }^{[13]}$ and Ni-Co ${ }^{[9,25]}$

from 1.4 to $2.4 \mathrm{GPa}$. A likely reason for the scatter is phase transformation. Pure Co and Co-rich alloys are highly unstable in the high-temperature FCC phase and when cooled (even at a rapid rate) to room temperature, transform to the HCP phase or a combination of FCC and HCP phases. ${ }^{[2,36]}$ In such scenario, the $H$ values obtained from nanoindentation would correspond to either of these phases, or both, and therefore result in observed scatter. Keeping this in view, data from Co-Fe and $\mathrm{Co}-\mathrm{Ni}$ belonging to the Co-rich end of the DCs $(c<\sim 2.5$ at. pct) are excluded from the Labusch plot in Figure 6. On the other hand, at higher concentrations, both $\mathrm{Fe}$ and $\mathrm{Ni}$ stabilize the FCC phase, which is retained to room temperature. ${ }^{[34]}$ 
Co-Mo, Co-Ni, and Co-Fe are observed to precisely obey the $c^{2 / 3}$ dependence of the Labusch model, while the SSH ability of $\mathrm{Pt}$ is seen to decrease with $c . k$ values from the linear fit show that Mo is an exceptionally strong SSH element $\left(938 \mathrm{MPa} /\right.$ at. pct $\left.^{2 / 3}\right)$, which is followed by strong SSH by $\mathrm{Pt}\left(479 \mathrm{MPa} / \mathrm{at} . \mathrm{pct}^{2 / 3}\right)$. The SSH potencies can be explained based on the large $\varepsilon$ values of 1.83 and 1.53 for $\mathrm{Co}-\mathrm{Mo}$ and $\mathrm{Co}-\mathrm{Pt}$, respectively, arising from their large $\Delta r$ and $\Delta E$ values. Note that the $k$ values obtained here correspond to FCC solid-solutions.

\section{E. Comparison of Ni and Co Binary FCC Solid-Solutions}

While the elastic misfit $\varepsilon$ values (Table I) are found to be similar for: $\mathrm{Ni}-\mathrm{Co}$ and $\mathrm{Co}-\mathrm{Ni}, \mathrm{Ni}-\mathrm{Fe}$ and $\mathrm{Co}-\mathrm{Fe}$, $\mathrm{Ni}-\mathrm{Pt}$ and $\mathrm{Co}-\mathrm{Pt}$, and $\mathrm{Ni}-\mathrm{Mo}$ and $\mathrm{Co}-\mathrm{Mo}$, the $k$ values are significantly different. This is shown in Figure 7, where, $k$ for each solvent-solute combination is plotted against the corresponding $\varepsilon^{4 / 3}$. Evidently, the addition of a specific solute to Co results in greater SSH than its addition to Ni. The effect of solvent on $\mathrm{SSH}$ coefficient is captured by the parameter $A$ in the model (Eq. [1]) through $k=A \varepsilon^{4 / 3}$. Indeed, $\mathrm{Co}$ and $\mathrm{Ni}$ solid-solutions follow linear (but distinct) relation between $k$ and $\varepsilon^{4 / 3}$ (Figure 7). However, for similar atomic sizes and modulus between the solvent and solute, i.e., $\varepsilon \rightarrow 0, k$ is expected to be negligible. While this holds for $\mathrm{Ni}$ (i.e., $\mathrm{Ni}-\mathrm{Co}$ ), a moderately high $k$ is observed for Co (i.e., $\mathrm{Co}-\mathrm{Ni}$ ), indicating that the elastic interactions alone do not explain SSH in Co. The values of parameter $A$ are obtained as 327 for Co solid-solution and $142 \mathrm{MPa} /$ at. $\mathrm{pct}^{2 / 3}$ for Ni solid-solution, with the standard error (of regression slope) in the respective values being 142 and $81 \mathrm{MPa} /$ at. $\mathrm{pct}^{2 / 3}$.

The additional contribution to SSH can be attributed to the low SFE of Co. As mentioned in Section IV-A, the slip misfit $\varepsilon_{S}{ }^{[28]}$ can be considered as an added contribution to $\varepsilon$. Assuming a linear variation in SFE for the sake of argument, $\varepsilon_{s}$ can be written as $\frac{\Delta \gamma}{\gamma}$; here, $\Delta \gamma$ is the difference between SFEs of FCC Ni (120 to $\left.130 \mathrm{~mJ} / \mathrm{m}^{2[37]}\right)$ and FCC Co $\left(\sim 10 \mathrm{~mJ} / \mathrm{m}^{2[38]}\right)$, and $\gamma$ is the SFE of the solvent. Thus, it can be seen that $\varepsilon_{\mathrm{S}}$ could provide a significant contribution to $k$ for Co (due to the low value of $\gamma$ ), suggesting a possible explanation for the difference between the $k$ values of $\mathrm{Co}$ and $\mathrm{Ni}$ alloys containing the same solute.

While linear trends are observed for $\mathrm{Ni}$ and $\mathrm{Co}$ solid-solutions in Figure 7, a positive deviation is seen for $\mathrm{Ni}-\mathrm{Mo}$, Ni-Ta, ${ }^{[9]}$ and Co-Mo. A similar behavior was found by Mishima et al. ${ }^{[31]}$ with $\mathrm{Mo}, \mathrm{Ta}, \mathrm{Nb}$, and $\mathrm{Zr}$ exhibiting a positive deviation from the linear fit to $\mathrm{Ni}$ binary solid-solution data (obtained from macroscopic testing). The deviation was attributed to contributions from electronic effects in transition metal solid-solutions. ${ }^{[31,39]}$ Also, solvent-solute combinations with a large difference in valence electrons are found to have a greater effect on SFE, ${ }^{[29]}$ and the occurrence of SFs is found to be more pronounced in alloys with larger atomic number difference. ${ }^{[35]}$ Thus, an additional contribution over the elastic effects, leading to higher values of $k$, can be expected. The effect is also pronounced for Co due to its low SFE, as observed in Figure 7.

The SSH behaviors evaluated in the present study are compared in Table I with literature studies employing similar DC-NI approach. For this, the $H-c$ data of $\mathrm{Ni}$ solid-solutions in References 9, 13, and 24 were analyzed (see Figure S2 in Supplementary Material) using the Labusch model to obtain the literature values of $k$. A generally good agreement is observed, indicating reproducibility of SSH behavior evaluated using the DC-NI approach. Inconsistencies for Pt-Ni (Section IV-B) and Ni-Mo are found, which are probably due to the difference in the modes of heat treatments employed and the indentation size effect. ${ }^{[24]}$

\section{CONCLUSIONS}

Solid-solution hardening ( $\mathrm{SSH}$ ) in certain $\mathrm{Fe}, \mathrm{Pt}, \mathrm{Co}$, and $\mathrm{Ni}$ binary alloys was revisited using a rapid combinatorial approach involving the diffusion couple (DC) method and the nanoindentation technique (NI). SSH data at large concentrations were evaluated within the framework of the Labusch model and assuming elastic contributions. SSH behaviors observed using the DC-NI approach were found to agree qualitatively with other studies employing conventional methods. SSH coefficients, $k$, were obtained by employing the Labusch fit to empirical data, and the $k$ values for $\mathrm{Ni}$ solid-solutions were found to be largely consistent with the literature on similar DC-NI approach. The linear Labusch dependence of hardness with $c^{2 / 3}$ was found to be generally obeyed at large concentrations. However, elastic contributions were found to be insufficient to explain SSH in Co solid-solutions and solid-solutions containing $\mathrm{Co}$ and $\mathrm{Mo}$, and contribution from stacking faults were identified as a possible additional contribution to SSH. Overall, the present study shows good reproducibility in studying SSH using the DC-NI approach, which could find potential utility in studying SSH in alloys of large concentrations like high-entropy alloys.

\section{ACKNOWLEDGMENTS}

The authors are grateful to Professor Aloke Paul for his expert guidance on the diffusion couple experiments. UR acknowledges the financial support to him from the Department of Science and Technology, Government of India through the award of the J.C. Bose National Fellowship.

\section{ELECTRONIC SUPPLEMENTARY MATERIAL}

The online version of this article (doi:10.1007/ s11661-017-4250-3) contains supplementary material, which is available to authorized users. 


\section{REFERENCES}

1. J.C. Fisher: Acta Metall., 1954, vol. 2, pp. 9-10.

2. R.L. Fleischer: Acta Metall., 1963, vol. 11, pp. 203-09.

3. T. Suzuki: Jpn. J. Appl. Phys., 1981, vol. 20, pp. 449-62.

4. M.Z. Butt and P. Feltham: J. Mater. Sci., 1993, vol. 28, pp. 2557-76.

5. D.B. Miracle and O.N. Senkov: Acta Mater., 2017, vol. 122, pp. $448-511$.

6. J.-C. Zhao, M.R. Jackson, L.A. Peluso, and L.N. Brewer: JOM, 2002, vol. 54, pp. 42-45.

7. J.-C. Zhao, X. Zheng, and D.G. Cahill: Mater. Today, 2005, vol. 8, pp. $28-37$

8. J.-C. Zhao: Prog. Mater. Sci., 2006, vol. 51, pp. 557-631.

9. O. Franke, K. Durst, and M. Göken: J. Mater. Res., 2009, vol. 24, pp. 1127-34.

10. V.D. Divya, S.S.K. Balam, U. Ramamurty, and A. Paul: Scr. Mater., 2010, vol. 62, pp. 621-24.

11. V.D. Divya, U. Ramamurty, and A. Paul: Philos. Mag., 2013, vol. 93, pp. 2190-2206.

12. V.V. Shastry, V.D. Divya, M.A. Azeem, A. Paul, D. Dye, and U. Ramamurty: Acta Mater., 2013, vol. 61, pp. 5735-42.

13. J-C Zhao: J. Mater. Res., 2001, vol. 16, pp. 1565-68.

14. W.C. Oliver and G.M. Pharr: J. Mater. Res., 2011, vol. 7, pp. 1564-83.

15. R. Labusch: Phys. Status Solid., 1970, vol. 41, pp. 659-69.

16. G.P.M. Leyson and W.A. Curtin: Philos. Mag., 2013, vol. 93, pp. $2428-44$.

17. C. Varvenne, G.P.M. Leyson, M. Ghazisaeidi, and W.A. Curtin: Acta Mater., 2017, vol. 124, pp. 660-83.

18. L.A. Gypen and A. Deruyttere: Scr. Metall., 1981, vol. 15, pp. 815-20.

19. H.W. King: J. Mater. Sci., 1966, vol. 1, pp. 79-90.

20. W.F. Gale and T.C. Totemeier: Smithels Metals Reference Book, 8th ed., Butterworth-Heinemann, Oxford, 2004.
21. V.D. Divya, U. Ramamurty, and A. Paul: Intermetallics, 2010, vol. 18, pp. 259-66.

22. W.B. Pearson and G.H. Vineyard: Phys. Today, 1958, vol. 11, p. 36.

23. W.C. Leslie: Metall. Trans., 1972, vol. 3, pp. 5-26.

24. K. Durst, O. Franke, A. Böhner, and M. Göken: Acta Mater., 2007, vol. 55 , pp. $6825-33$.

25. C.K.L. Davies, V. Sagar, and R.N. Stevens: Acta Metall., 1973, vol. 21, pp. 1343-52.

26. E.A. Owen and Y.H. Liu: J. Iron Steel Inst., 1949, vol. 163, p. 132.

27. L. Kaufman and M. Cohen: Prog. Met. Phys., 1958, vol. 7, pp. 165-246.

28. J.A. Yasi, L.G. Hector, and D.R. Trinkle: Acta Mater., 2010, vol. 58 , pp. 5704-13.

29. K.R. Limmer, J.E. Medvedeva, D.C. Van Aken, and N.I. Medvedeva: Comput. Mater. Sci., 2015, vol. 99, pp. 253-55.

30. T. Murakami, R. Sahara, D. Harako, M. Akiba, T. Narushima, and C. Ouchi: Mater. Trans., 2008, vol. 49, pp. 538-47.

31. Y. Mishima, S. Ochiai, N. Hamao, M. Yodogawa, and T. Suzuki: Trans. Jpn. Inst. Met., 1986, vol. 27, pp. 656-64.

32. G. Choi, T. Shinoda, Y. Mishima, and T. Suzuki: ISIJ Int., 1990, vol. 30 , pp. $248-54$

33. H.A. Roth, C.L. Davis, and R.C. Thomson: Metall. Mater. Trans. A, 1997, vol. 28A, pp. 1329-35.

34. T.C. Tisone: Acta Metall., 1973, vol. 21, pp. 229-36.

35. L. Deléhouzée and A. Deruyttere: Acta Metall., 1967, vol. 15, pp. $727-34$

36. J.B. Newkirk and A.H. Geisler: Acta Metall., 1953, vol. 1, pp. $456-57$.

37. C.B. Carter and S.M. Holmes: Philos. Mag., 2006, vol. 35, pp. 1161-72.

38. W. Charnock and J. Nutting: Met. Sci. J., 2013, vol. 1, pp. 12327.

39. T. Shinoda, K. Masuda-Jindo, Y. Mishima, and T. Suzuki: Phys. Rev. B, 1987, vol. 35, pp. 2155-61. 